\title{
Organic Light Emitting Diodes: Device Physics and Effect of Ambience on Performance Parameters
}

\author{
T.A. Shahul Hameed ${ }^{1}$, P. Predeep ${ }^{1}$ and M.R. Baiju ${ }^{2}$ \\ ${ }^{1}$ Laboratory for Unconventional Electronics and Photonics, National Institute of \\ Technology, Calicut, Kerala, \\ ${ }^{2}$ Department of Electronics and Communication, College of Engineering, \\ Trivandrum, Kerala, \\ India
}

\section{Introduction}

Research in Organic Light Emitting Diode (OLED) displays has been attaining greater momentum for the last two decades obviously due to their capacity to form flexible (J. H. Burroughes et al, 1990) multi color displays. Their potential advantages include easy processing, robustness and inexpensive foundry (G.Yu \& A.J.Heeger, 1997) compared to inorganic counterparts. In fact, this new comer in display is rapidly moving from fundamental research into industrial product, throwing many new challenges (J. Dane and J.Gao, 2004; G. Dennler et al, 2006) like degradation and lifetime. In order to design suitable structures for application specific displays, the studies pertaining to the device physics and models are essentially important. Such studies will lead to the development of accurate and reliable models of performance, design optimization, integration with existing platforms, design of silicon driver circuitry and prevention of device degradation. More over, a clear understanding on the device physics (W.Brutting et al, 2001) is necessary for optimizing the electrical properties including balanced carrier injection (J.C.Scott et al, 1997: A.Benor et al., 2010) and the location of the emission in the device. The degradation (J.C.Scott et al, 1996; J. Dane and J.Gao, 2004) of the device is primarily caused by the moisture, which poses questions to the reliability and life of this promising display. How the device responds to different temperature ambience (T.W.Lee and O.Park, 2000) also attracts attention of researchers since its applications at cryogenic temperature are yet to be explored. The basic device physics and modeling philosophies based on the mathematical formulations of its physical behavior are revisited in this article. Also it reviews the prominent ambient studies and the efforts to enhance the reliability of the device by new fabrication methods with inexpensive ways of encapsulation, making it suitable for long life display applications.

\section{Principle and physics of organic LEDs}

\subsection{Device structure, principle}

The simplest structure of OLED is shown in fig 1. The Tris(8-hydroxyquinolinato) aluminium (Alq3) is an evaporated emissive layer on the top of spun cast hole transport 
layer Poly-(3,4-ethyhylene dioxythiophene):poly-(styrenesulphonate) (PEDOT:PSS). Indium Tin Oxide (ITO) and aluminium are the anode and cathode respectively. Charge injection, transport and recombination (I.H.Campbell et al,1996) occur in the light emitting conductive layer of organic light emitting diodes and its features influence efficiency and color of emission from the device. Besides the characteristics of light emitting organic layer, interface interactions (P.S.Davids et al, 1996) of this layer with other layers in OLED play important role in defining the characteristics of the display. There have been innumerable studies on different aspects of PEDOT: PSS (L.S.Roman et al,1999;S.Alem et al,2004) enhancing the performance of photo cells and light emitting diodes. In practical implementations, more layers for carrier injection and transport are normally incorporated.

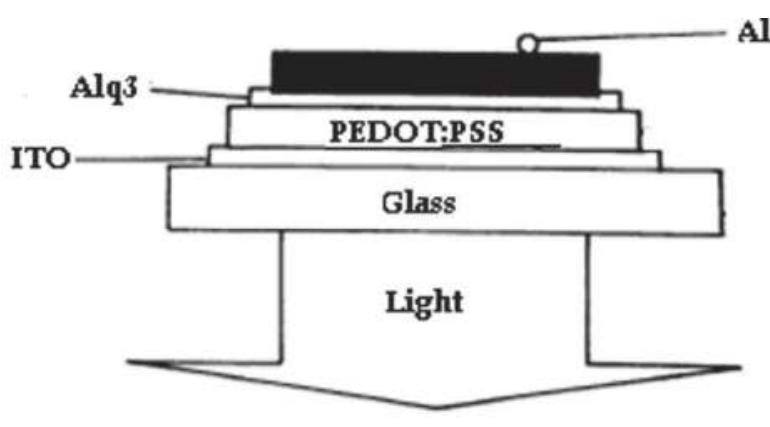

Fig. 1. Structure of Organic Light Emitting Diode.

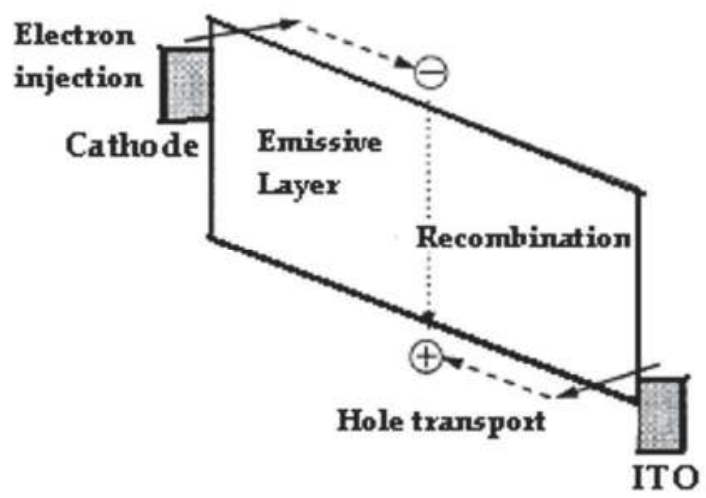

Fig. 2. Injection, Transport and Recombination in PLED[15].

In Polymer Light Emitting Diodes(PLED), conducting polymers like Poly (2-methoxy, 5-(2ethylhexoxy)-1, 4-phenylene-vinylene (MEH- PPV) are used as the emissive layer in which dual carrier injection takes place (Fig. 2). Electrons are injected from cathode to the LUMO of the polymer and holes are injected from anode to HOMO of the conducting polymer and they recombine radiatively within the polymer to give off light (Y.Cao et al,1997). The fabrication of the device is easy through spin casting of the carrier transport layer and Electro Luminescent layer (MEH-PPV) for thickness in $A^{\circ}$ range. 


\subsection{Device physics}

For OLEDs, it is more often a practice to follow many concepts derived from inorganic semiconductor physics. In fact, most of the organic materials used in LEDs form disordered amorphous films without forming crystal lattice and hence the mechanisms used for molecular crystals cannot be extended. Detailed study on device physics of organic diodes based on aromatic amines (TPD) and aluminium chelate complex (Alq) was carried out by many research groups (W.Brutting et al,2001).Basic steps in electroluminescence are shown in fig. 3 where charge carrier injection, transport, exciton formation and recombination are accounted in presence of built-in potential. Built-in potential(Vbi) across the organic layers is due to the different work functions between anode and cathode (I.H.Campbell et al,1996).

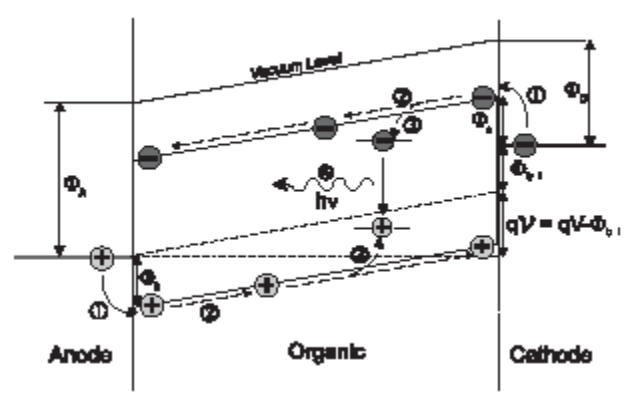

Fig. 3. Basic Steps of Electroluminescence with Energy Band[4].

Built-in potential (Vbi) found out by photovoltaic nulling method, where OLED is illuminated and an external voltage is applied till photocurrent is equal to dark current (J.C.Scott et al,2000). Its physical significance is that it reduces the applied external voltage $\mathrm{V}$ such that a net drift current in forward bias direction can only be achieved if $\mathrm{V}$ exceeds built in voltage.Carrier injection is described by Fowler-Nordheim tunneling or RichardsonSchottky thermionic emission, described by the equations

$$
\begin{aligned}
& j_{F N}=\frac{A^{*} q^{2} F^{2}}{\phi_{B} \alpha^{2} K_{B}{ }^{2}} \exp \left(-\frac{2 \alpha \phi_{B}{ }^{3 / 2}}{3 q F}\right) \\
& j_{R S}=A^{*} T^{2} \exp \left(-\frac{\phi_{B}-\beta_{R S} \sqrt{F}}{K_{B} T}\right)
\end{aligned}
$$

The current is either space charge limited (SCLC) or trap charge limited (TCLC).The recombination process in OLED has been described by Langevin theory because it is based on a diffusive motion of positive and negative carriers in the attractive mutual Coulomb field. To be more clear, the recombination constant $(\mathrm{R})$ is proportional to the carrier mobility (W.Brutting et al,2000).

$$
R=\left[q / \varepsilon \varepsilon_{0}\right]\left[\mu_{h}+\mu_{e}\right]
$$

Apart from the discussion on the dependence of current on voltage and temperature, the current has a direct dependence on the thickness of the organic layer and it was observed that thinner the device better will be the current output. Similar observations were also 
made by the group on J-V and luminance characteristics of ITO/TPD/AlQ/Ca hetero junction devices for different organic layer thickness. The thickness dependence of current at room temperature leads to the inference that the electron current in Alq device is predominantly space charge limited with a field dependent charge carrier mobility and that trapping in energetically distributed states is additionally involved at low voltage and especially for thick layers. The temperature dependence of current in $\mathrm{Al} / \mathrm{Alq} / \mathrm{Ca}$ device (from $120 \mathrm{~K}$ to $340 \mathrm{~K}$ ) indicates that device is having a less turn-on current at higher temperature and recombination in OLED to be bimolecular process following the Langevin theory. The mathematical analysis of the device, considering traps and temperature has been a new approach in device physics.

Towards the search of highly efficient device, the combining of Alq and NPB, with a thickness of $60 \mathrm{~nm}$ for the Alq layer has been determined to yield higher quantum efficiency whereas thickness variation of NPB layer doesn't show any measurable effect.

The field and temperature dependence of the electron mobility in Alq leads to the delay equation (W.Brutting et al,2000) as

$$
t_{d}=\frac{d}{\mu F}
$$

where

$$
F=\frac{V-V_{b i}}{d} .
$$

The behavior of hopping transport in disordered organic solids has been better explained by Gaussian Disorder Model (H.Bassler,1993). The quantitative model for device capacitance with an equivalent circuit of hetero layer device gives more insight into interfacial charges and electric field distribution in hetero layer devices.

The transport behavior in polymer semiconductor has been a matter of active debate since many theories were put forwarded by different groups. Charge transport is not a coherent motion of carriers in well defined bands - it is a stochastic process of hopping between delocalized states, which leads to low carrier mobilities $\left(\mu<<1 \mathrm{~cm}^{2} / V s\right)$ (W. Brutting et al,1999). Trap free limit for dual carrier device was studied by Bozano et al,1999. Space charge limited current was observed above moderate voltages $(>4 \mathrm{~V})$, while zero field electron mobility is an order of magnitude lower than hole mobility. Balanced carrier injection is one of the pre requisites for the optimal operation of single layer PLEDs. Balanced carrier transport implies that injected electrons and holes have same drift mobilities. In fact, it is difficult to achieve in single layer devices due to the predominance of one of the carriers and hence bi-layer devices are used to circumvent the problem. ITO/PPV/TPD: PC/Al devices fabricated where ITO/PPV is an ideal hole injecting contact for the trap-free MDP TPD: PC. Here ITO/PPV contact acts as an infinite, non depletable charge reservoir, which is able to satisfy the demand of the TPD: PC layer under trap-free space-charge-limited (TFSCL) conditions (H.Antoniadis et al,1994). Trap free space charge limited current (TFSL) [L.Bozano et al,1999) can be expressed as

$$
J_{T F S L}=\frac{9}{8} \varepsilon \varepsilon_{0} \mu E^{2} / d
$$


where $\varepsilon_{0}$ is the permittivity of vacuum, $\varepsilon$ is the permittivity of the polymer, $\mu$ is the mobility of holes in trap free polymer, $\mathrm{d}$ is inter electrode distance(M. A. Lampert and P. Mark ,1970). Trapping is relatively severe at low electric fields and in thick PPV layers. At high electric fields, trapping is minimized even for thick PPV layers.

The carrier drift distance $x$ at a given electric field $\mathrm{E}$ before trapping occurs is given by $x=\mu \tau E$ where $\tau$ is the trapping time. The electron deep trapping product $\mu \tau$ determines the average carrier range per applied electric field before they get immobilized in deep traps. It is imperative that the difference in $\mu \tau$ values of electrons and holes in PPV $\left(10^{-12}\right.$ and $10^{-9} \mathrm{~cm}^{2} / v$ respectively) reflects their discrepancy in transport. In fact, not the structure of PPV contributes to this difference, but oxygen related impurities in PPV (P.K. Konstadinidis et al,1994) with strong electron accepting character and reduction potential lower than PPV may act as the predominant electron traps and limit the range of electrons. The study of temperature dependence of current density versus electric field for single carrier (both electron dominated and hole dominated) and dual carrier devices at temperatures $200 \mathrm{~K}$ and $300 \mathrm{~K}$ exhibits interesting results (L.Bozano et al,1999). In both temperatures, the reduction in space charge due to neutralization contributes to significant enhancement in current density in dual carrier devices. Also it was deduced that the electric field dependence of the mobility is significantly stronger for electrons than for holes. The electric field coefficient $\gamma$ is related to temperature as per the empirical relation $\gamma=\left(1 / k T-1 / k T_{0}\right) B$ where $B$ and $T_{0}$ are constants (W.D.Gill,1972). In MEH-PPV devices, charge balance will be improved by cooling which in turn leads to enhanced quantum efficiency. By adjusting barrier heights, at the level of $0.1 \mathrm{eV}$, quantum efficiency close to theoretical maximum can be achieved. In order to limit the space charge effects and hence to enhance the performance in terms of current density, the intrinsic carrier mobility to be taken care by modifying dielectric constant or electrically pulsing the device at an interval greater than recombination time. The other means of improvisation is aligning of polymer backbone, but such efforts may lead to quenching (L.Bozano et al,1998)

\subsection{Device models}

Device modeling is useful in many ways like optimization of design, integration with existing tools, prediction of problems in process control and better understanding of degradation mechanism. By modeling PLEDs current-voltage -luminance behavior, with which quantum and power efficiencies can be analytically seen, this in turn normally has to be subjected to experimental validation.

Both band based models and exciton based models were proposed to explain the electronic structure and operation of polymer devices. Out of the two, there are more supportive arguments for band based model. I.D.Parker examined (I.D.Parker,1994) the factors that control carrier injection with a particular reference to tunneling, by experimenting on ITO/MEH-PPV/Ca device. The thickness dependability of current density with respect to bias and field strength are shown in fig. 4 and 5 respectively. It is obvious from these figures that the device operating voltage shall be reduced by reducing the polymer thickness. The field dependence of $\mathrm{I}-\mathrm{V}$ behavior points to the tunneling model of carrier injection, in which carriers are field emitted through a barrier at electrode/ polymer interface (fig.4). 


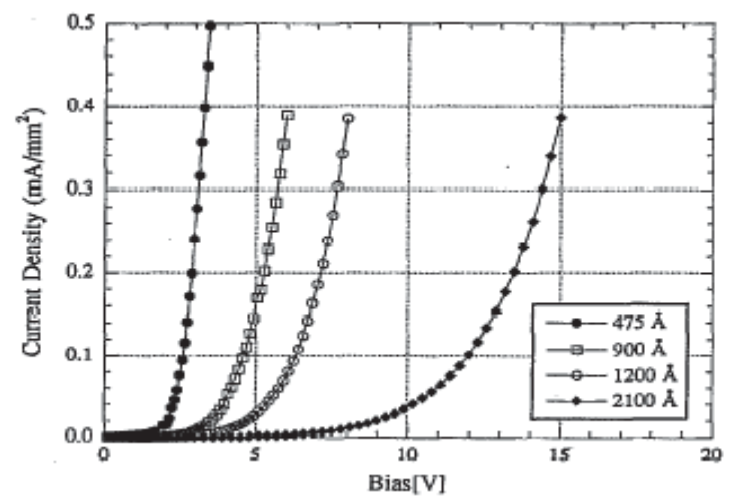

Fig. 4. Thickness Dependence of the I-V Characteristics in ITO/MEH-PPV/Ca Device (I.D.Parker,1994).

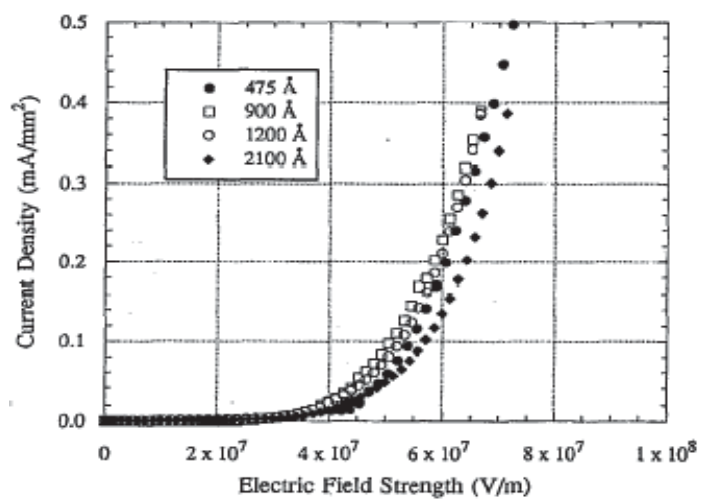

Fig. 5. Field $v$ Current Dependence for ITO/MEH-PPV/Ca Device ((I.D.Parker,1994).

For a clear understanding of the device physics and models, it is customary to fabricate single carrier and dual carrier devices. On replacing $\mathrm{Ca}$, having low work function $(2.9 \mathrm{eV})$ with higher work function metals like In $(4.2 \mathrm{eV}), \mathrm{Au}(5.2 \mathrm{eV})$, hole only devices can be made. This increases the offset between Fermi energy of cathode and LUMO of polymer which causes a substantial reduction in injected electrons and holes become dominant carriers. It is apparent that the external quantum efficiency reduces in single carrier devices. The current characteristics show only a slight dependence with temperature which is predicted by Fowler-Nordheim tunneling.

$$
I \propto F^{2} \exp \left(\frac{-k}{F}\right)
$$

where $\mathrm{F}$ is the field strength The constant $\mathrm{k}$ is defined by

$$
k=\frac{8 \pi \sqrt{2 m^{*} \phi^{3 / 2}}}{3 q h}
$$


where $\phi$ is the barrier height and $m^{*}$ is the effective mass of the holes(S.M.Sze,1981).

A rigid band model better explains experimental results where holes and electrons tunnel into the polymer when applied electric field tilts the polymer bands to present sufficiently thin barriers. Fig. 6 clearly indicates how this model envisages tunneling of holes.

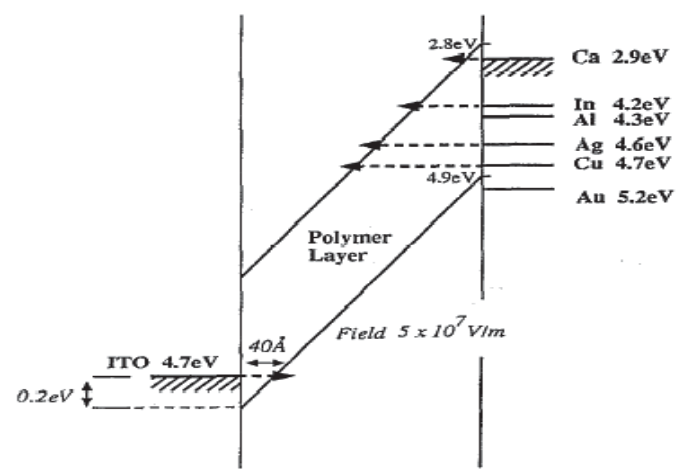

Fig. 6. Band Diagram (in Forward Bias) for Model, indicating positions of Fermi Level for different electrode materials (I.D.Parker,1994).

From the band based model and characterization, the improvements in device performance was suggested by I.D. Parker. Of the devices he made, ITO/MEH-PPV/Ca devices exhibit better results due to the reasons explained elsewhere. The device turn - on happens at a flat band condition and it is in fact the voltage required to reach the flat-band condition and it depends on the band gap of the polymer and work-function of electrodes. The operating voltage of the device is sensitive to barrier height whereas the turn-on voltage is not.

From the equations mentioned before, an approximation for the current can be made as

$$
I \propto \exp \left(-\frac{\gamma \phi^{2}}{V}\right)
$$

where $\mathrm{V}$ is the applied voltage and $\phi$ is the barrier height. This prediction of barrier height dependence of operating voltage has been supported by experimental credentials.

Efficiency of the device is a function of current density due to minority carriers, increasing barrier height leads to an exponential decrease in current and efficiency, which is shown in fig.7.Parker had suggested the suitable combination of electrode materials and polymers so that low turn-on voltage and operating voltage can be achieved.

J.C.Scott et al(J.C.Scott et al,2000) contributed to unveil the phenomena like built in potential, charge transport, recombination and charge injection with a numerical model to calculate the recombination profile in single and multilayer structures. 'Essentially trap free' transport, Langevin mechanism for recombination and model of thermionic injection with Schottkey barrier at metal organic interface are the important features used by them. It is to be highlighted that charge trapping is neglected in the analysis and transport is described in terms of trap free space charge limited currents. Fowler-Nordheim mechanism was used to explain the injection, but by analytical methods and simulations, thermionic injection ( G.G. Malliaras ,1998) is said to best suit for explaining the injection in organic diodes. 


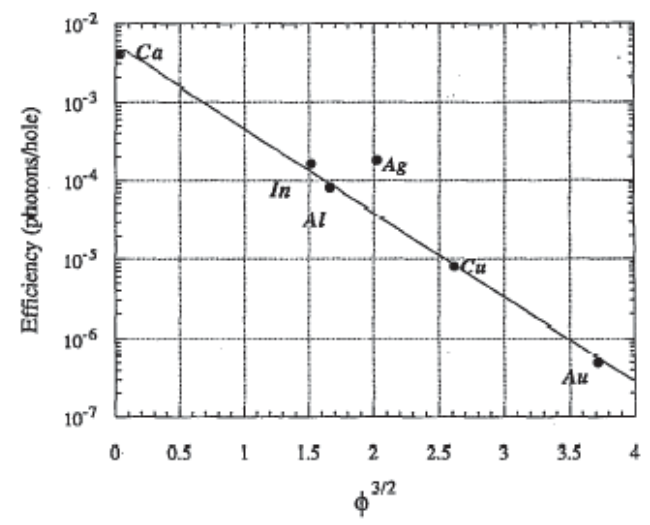

Fig. 7. Device Efficiency $v$ (Barrier Height) ${ }^{3 / 2}$ [I.D.Parker,1994).

There are remarkable efforts (P.W.M.Blom \& Marc J.M,1998) in characterization and modeling of polymer light emitting diodes. Their experiments on PPV devices, both single carrier and dual carrier devices, paved the way to the better understanding of mobility of electrons and holes. Electron only devices are fabricated by a PPV layer sandwiched between two Ca electrodes whereas hole only devices with an evaporated Au on top. For hole only devices, current density depends quadratically on voltage.

$$
J=\frac{9}{8} \varepsilon_{0} \varepsilon_{r} \mu_{p} \frac{V^{2}}{L^{3}}
$$

where $\mu_{p}$ is hole mobility and $\mathrm{L}$ is the thickness of the device. Transport properties of the single carrier devices are described in detail with analytical expressions. Hole only device is having effect of space charge holes and electron only devices show trapping of electrons. For double carrier device, two additional phenomenon becomes important-recombination and charge neutralization. Recombination is bimolecular since its rate is directly proportional to electron and hole concentration. Without traps and field dependent mobility, the current in double carrier device is

$$
J=\left(\frac{9 \pi}{8}\right)^{1 / 2} \varepsilon_{o} \varepsilon_{r}\left(\frac{2 q \mu_{p} \mu_{n}\left(\mu_{p}+\mu_{n}\right)}{\varepsilon_{o} \varepsilon_{r} B}\right)^{1 / 2} \frac{V^{2}}{L^{3}}
$$

where B is bimolecular recombination constant. (P.W.M.Blom \& Marc J.M,1998).

In PLEDs, conversion efficiency is dependent on applied voltage whereas in conventional LEDs, it is not. Temperature dependence of charge transport in PLEDs is investigated by performing J-V measurements on hole only and double carrier devices. Carrier transport strongly dependent on temperature (P.W.M.Blom et al, 1997) and the fig.8 explains the variation of current density with respect to applied voltage for different temperature.

Also, the plot of bimolecular recombination constant B for different temperatures (fig.9) sheds light into the fact that recombination is Langevin type [31] and mathematically it is expressed in terms of mobility

$$
B=\frac{e}{\varepsilon_{0} \varepsilon r}\left(\mu_{n}+\mu_{p}\right)
$$




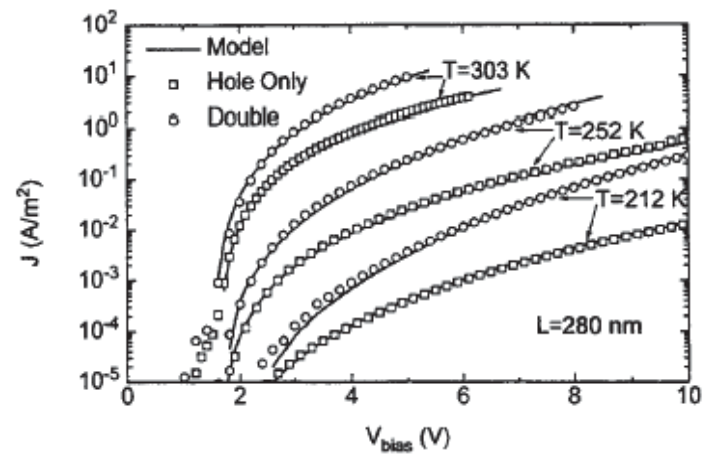

Fig. 8. Experimental and Calculated (Solid lines) J-V characteristics in hole only (squares) and double carrier (circle) for different thickness (P.W.M.Blom \& Marc J.M,1998).

The enhancement of maximum conversion efficiency is by decreasing non radiative recombination and by use of electron transport layer which shifts recombination zone away from metallic cathode.

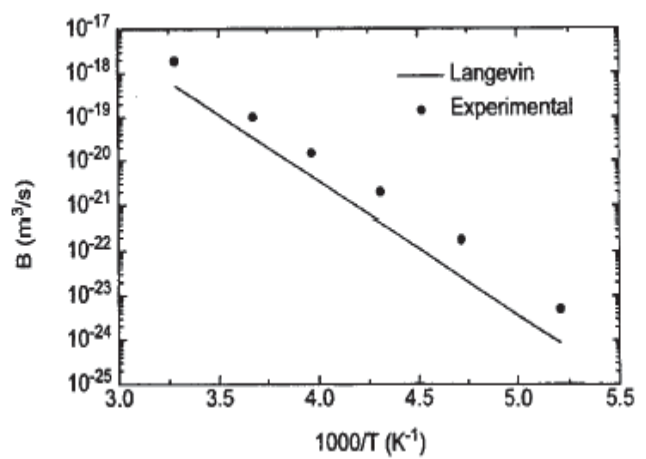

Fig. 9. Temperature Dependence of Bimolecular Recombination Constant (P.W.M.Blom \& Marc J.M,1998).

Device model based on Poisson's equation and conservation of charges was more a traditional presenattion (Y.Kawabe et al,1998) in organic electronic devices. By assuming that recombination rate is proportional to collision cross section A, electric field, sum of mobility values of electrons and holes and the product of carrier densities, charge conservation equation has been rewritten as

$$
\frac{d J_{h, e(x)}}{d x}= \pm A\left(\mu_{h}+\mu_{e}\right) E(x) n_{h}(x) n_{e}(x)
$$

where + and - signs indicate electron and hole currents.

By conservation law of the total current

$$
J_{h(x)}+J_{e(x)}=e E(x) n_{h}(x) \mu_{h}+e E(x) n_{e}(x) \mu_{e}=J_{0},
$$


with the boundary conditions given by current injection at both electrodes (Y.Kawabe et al,1998) .

Besides, current density, relative quantum efficiency was calculated by the model equation

$$
\eta=\frac{J_{h(0)}-J_{h(d)}}{J_{0}}=\frac{J_{e(d)}-J_{e(0)}}{J_{0}}
$$

Here numerical values of the parameters are used to simulate $\mathrm{J}-\mathrm{V}$ and quantum efficiency characteristics .Two devices-one with semiconducting polymer (BEH-PPV) and the other with dye doped polymer $\left(P V K: A l Q_{3}\right)$ were fabricated by spin casting techniques and characterized. The results validate the model for the single layer devices and its suitability for complex devices is yet to be tested.

The model is having the advantages of incorporating charged traps as shown in equation below

$$
\frac{d E(x)}{d x}=\frac{e}{q}\left[n_{h}(x)-n_{e}(x) \pm n_{t}(x)\right]
$$

where \pm indicates positive ad negative charges respectively. This sends limelight to the causes of degradation process in real devices due to the accumulation of electrons in the vicinity of the cathode. The inferences include low barrier height for low voltage operation, high mobility for high brightness devices and low electron mobility confines the emission region near the cathode and should be avoided to prevent electrode quenching.

\section{Ambient studies of organic light emitting diodes}

The temperature dependence of current density versus bias voltage exhibits interesting results in organic light emitting diodes. The studies made on four sets of devices namely Device A: ITO/PEDOT-PSS/MEH-PPV/Al, Device B: ITO/PEDOT-PSS/MEH$\mathrm{PPV} / \mathrm{LiF} / \mathrm{Al}$, Device C: ITO/PEDOT-PSS/Alq3/Al and Device D: ITO/PEDOT$\mathrm{PSS} / \mathrm{Alq} 3 / \mathrm{LiF} / \mathrm{Al}$ show the effects of temperature variation in their performance. The OLEDs were fabricated on ITO coated glass of surface resistivity in the range of tens of ohms. The standard cleaning procedure (] W. H. Kim et al,2003) in deionized water, acetone and isopropyl alcohol were carried out. PEDOT:PSS and MEH:PPV were spun cast on ITO coated glass for polymer devices. For fabricating small molecule based OLEDs, Tris(8hydroxyquinolinato) aluminium (Alq3) was vacuum evaporated at $10^{-6}$ torr by physical vapor deposition. The buffer layer of $\mathrm{LiF}$ was also vacuum evaporated in the devices where such caps were used to enhance the injection of carriers. The metallic cathode was also vacuum evaporated in all the four sets of devices.The J-V characteristics were plotted by using a Keithley 2400 Source meter interfaced to a computer. Impedance versus frequency behavior was studied using Electrochemical workstation IM6 ex from Zahner, Germany. It also gives the plots of real versus imaginary impedances. The measurements from cryogenic temperature to room temperature were taken with the help of cryostat. The thickness of the evaporated as well as spun cast layers and refractive index of PEDOT:PSS film on ITO were measured by Sopra make Spectroscopic Ellipsometer. The luminance behavior was observed with the help of a fibre optic spectrometer Avantes. 


\subsection{Current density versus bias voltage}

The variation of current density with respect to the applied voltage explains the turn on phenomena of the device. Figures 10 and 11 show the J-V characteristics of devices A, B, C and $\mathrm{D}$ respectively at a temperature varying from very low value of $100 \mathrm{~K}$ to room temperature. The devices A and B are having MEH:PPV as the emissive layer and their J-V characteristics are shown in figure $10 \mathrm{a}$ and $10 \mathrm{~b}$ respectively. The devices $\mathrm{C}$ and $\mathrm{D}$ in which the emissive material is small molecule Alq3 exhibits a current variation as shown in figure $11 \mathrm{a}$ and $11 \mathrm{~b}$ respectively.
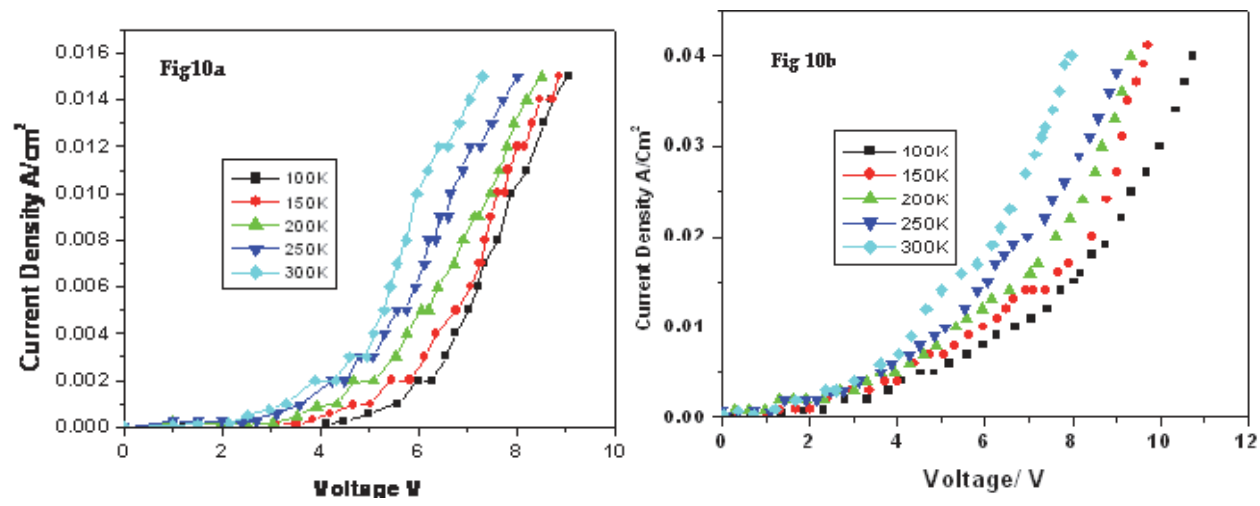

Fig. 10. JV characteristics of Device A and B at different temperatures.
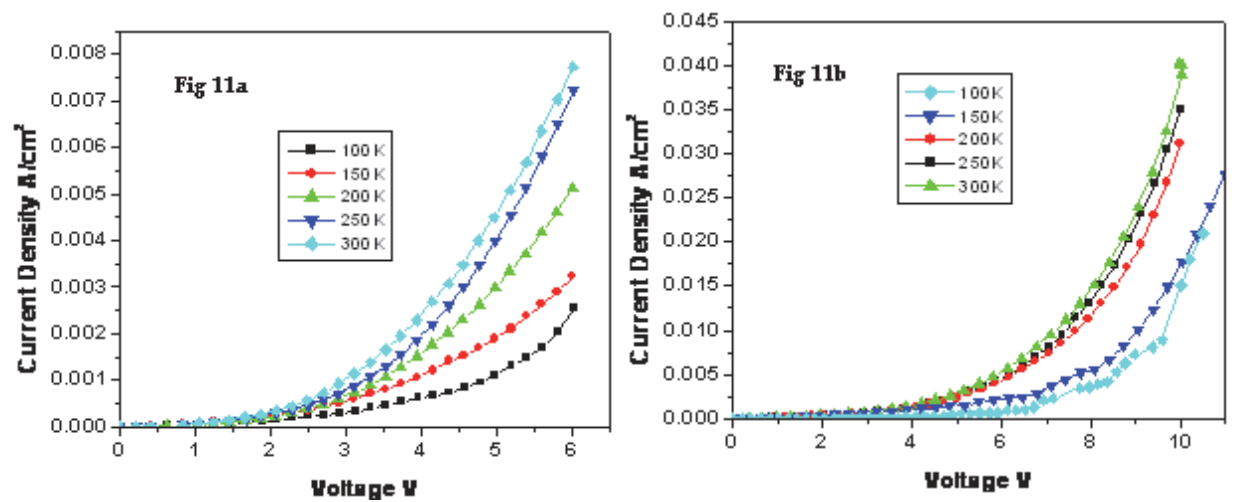

Fig. 11. J-V Characteristics of Device C and D at different temperatures.

The lowest voltage required [26] for the start of tunneling and hence the light emission is the 'turn on' voltage. At very small forward voltage, tunneling doest not occur and it begins at the flat band condition. In fact, 'flat band voltage' is the energy gap minus the two energy offsets. The turn on voltage is a function of the energy levels of the polymer and considered to be independent of the polymer thickness. The emission from the device starts to occur at a point where the current starts to increase rapidly when plotted in linear axis. This is the 'operating voltage' at which light emission becomes visible to the naked eye and it is a function of the thickness of the emissive layer. 
In the device A, 'turn on' happens at 4 volts at $100 \mathrm{~K}$ and it gradually comes down at every fall of $50 \mathrm{k}$ and finally it reaches 2.2 volts at $300 \mathrm{~K}$. For the device where LiF buffer layer (device B) is used to catalyze the carrier injection, 'turn on' occurs bit earlier than device A2.8 volts at $300 \mathrm{~K}$ and falls to 2.1 volts at $100 \mathrm{~K}$. It is obvious that the rate of this fall in device $\mathrm{A}$ is more than that of device $\mathrm{B}$. The operating voltage also experiences a similar shift due to the variation in temperature- 5.8 volts at $100 \mathrm{~K}$ to 3.9 volts at $300 \mathrm{~K}$ in device $\mathrm{A}$ and in the case of device B, it is 4 volts at $100 \mathrm{~K}$ to 2.9 volts at $300 \mathrm{~K}$. A similar variation can be seen in organic light emitting devices also where Alq3 is the emissive material.

In all the four sets of devices, it was observed that the 'turn on' occurs at smaller values of applied bias voltage in room temperature. As the temperature goes on decreasing, the turn on becomes slower and it becomes worst at the lowest temperature of $100 \mathrm{~K}$. At lower forward bias Fowler Nordheim tunneling contributes to the device current whereas at higher bias voltages, space charge limited current (SCLC) governs the current. The current density in dual carrier device is a direct function of the product and the sum of the mobilities of electrons and holes (P.W.M.Blom et al,1998), which is clear from the eqn.10.

$$
J=\left(\frac{9 \pi}{8}\right)^{1 / 2} \varepsilon_{o} \varepsilon_{r}\left(\frac{2 q \mu_{p} \mu_{n}\left(\mu_{p}+\mu_{n}\right)}{\varepsilon_{o} \varepsilon_{r} B}\right)^{1 / 2} \frac{V^{2}}{L^{3}}
$$

On increasing the recombination constant $B$, the neutralization decreases which brings down the current density. The mobilities at lower temperatures substantially come down which contribute to the slower 'turn on' process at lower temperatures.

\subsubsection{Impedance characteristics}

Impedance spectroscopy is a powerful tool (Shun-Chi Chang et al,2001) to investigate the behavior of OLEDs when applied with an alternating input having a frequency ranging from tens of hertz to several hundreds of kilohertz with a small ac input signal like $100 \mathrm{mV}$ peak to peak and this can be performed in the presence or absence of a superimposing DC voltage. The use of lower excitation voltage could assure the quasi-equilibrium condition needed to carry out such experiments and probe charged states in the bulk. Further, small ac voltage without a superimposing DC voltage would ensure clear separation of bulk effects from interfacial effects. By using spectroscopic investigations, real versus imaginary impedance can be derived which helps to evolve the electrical models of the device.

The equivalent circuit of OLED is normally represented by a series resistance with a parallel combination of resistance and capacitance in the case of single layer devices. More RC layers to be included when more layers are added in the device. This is normally deduced from the real and imaginary impedance obtained through impedance spectroscopy. The resistance and capacitance can be computed by fixing the points of series resistance (Rs) and the parallel resistance as shown in the figure 12. From the measurements of imaginary impedance ( $\left.Z^{\prime \prime}\right)$ the frequency corresponding to its maximum value can be equated as $\omega=$ $1 /(\mathrm{Rp} . \mathrm{Cp})$. From this equation value of $\mathrm{Cp}$ can be computed and the equivalent circuit is drawn as shown in figure 13. It is to be highlighted that when PEDOT:PSS is used as hole transport layer in organic or polymer devices, the impedance spectra resembles to that of a single layer device giving only one semicircle in the Cole-Cole plot or only one peak in the imaginary impedance measurements. In the real versus imaginary impedance plot (ColeCole plot), frequency is always an implicit variable. 


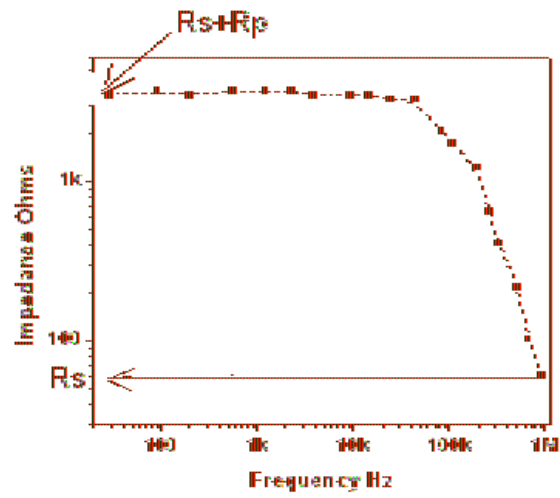

Fig. 12. Deducing equivalent circuit from impedance plots.

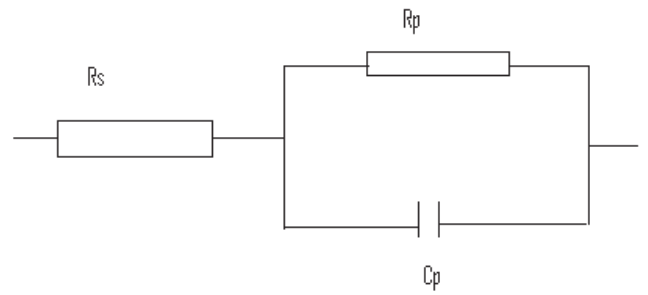

Fig. 13. Equivalent Circuit.

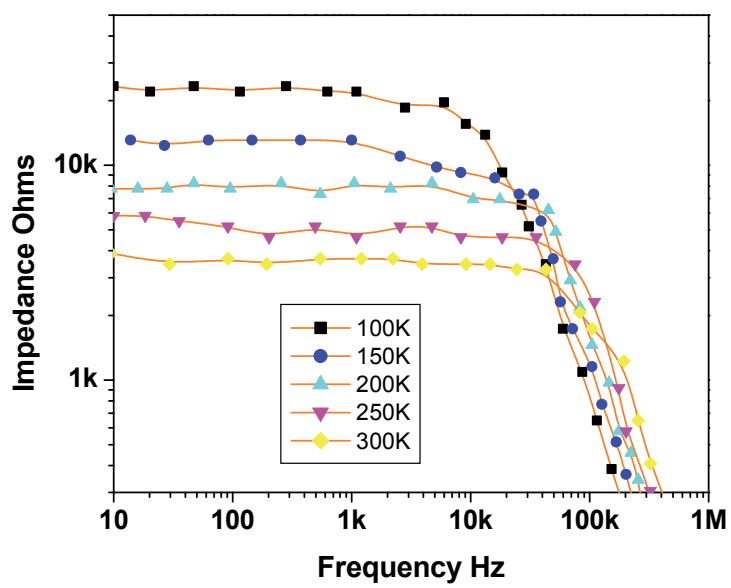

Fig. 14a. Impedance spectra of ITO/PEDOT:PSS/MEH:PPV/Al. 
The qualitative difference between the device behaviors when subjected to a small excitation of $100 \mathrm{mV}$ peak to peak with no superimposing DC voltages at different temperature is an interesting case to be analyzed. Figures 14 and 15 show the impedance spectra of the devices which use the polymer and small molecule electroluminescent layers in dual carrier injection devices. It is clear from the figure $14 \mathrm{a}$ and $14 \mathrm{~b}$ that the device in which a buffer layer of $\mathrm{LiF}$ is used (device $\mathrm{B}$ ) offers more impedance at the same frequency than the one without it (device A).

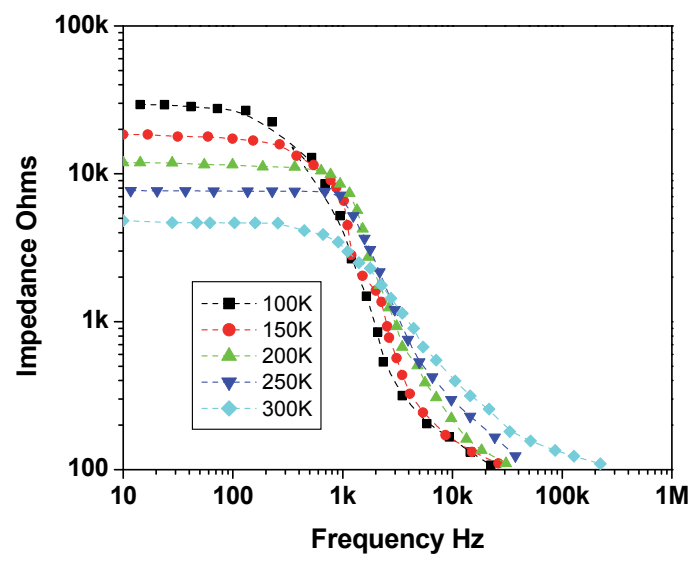

Fig. 14b. Impedance spectra of ITO/PEDOT:PSS/MEH:PPV/LiF/Al.

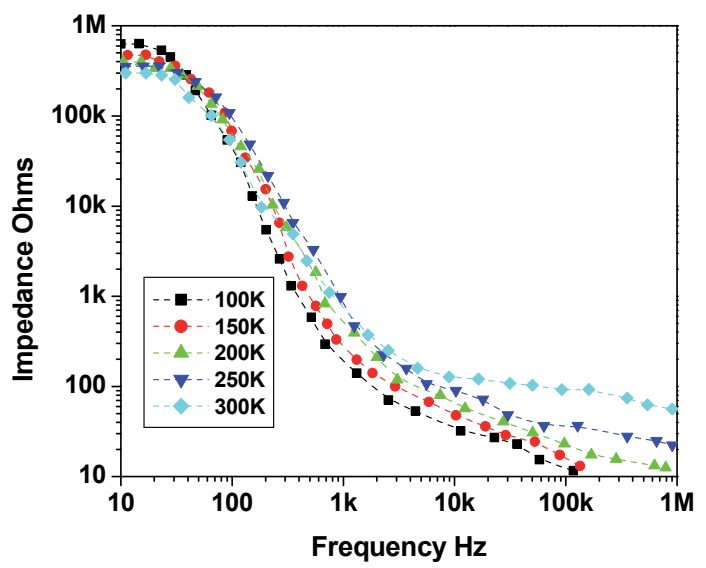

Fig. 15a. Impedance spectra of ITO/PEDOT:PSS/Alq3/Al. 


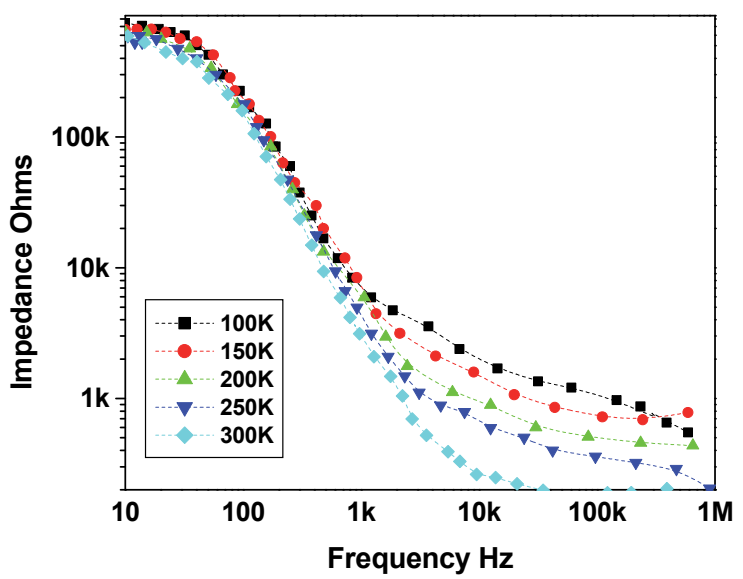

Fig. 15b. Impedance spectra of ITO/PEDOT:PSS/Alq3/LiF/Al.

In both cases, impedance remains high for higher value of frequency and it comes down as temperature is lowered. The impedance falls at lower frequencies in device B than A.

In the case of the devices which use small molecule Alq3 as emissive layer exhibits a higher impedance than that of MEH:PPV device in identical thickness of the layers. Here in low frequency regime of the spectra, the impedance remains constant for a smaller span of frequencies than that of the polymer devices. It is worth mentioning that the organic light emitting device which does not use a buffer layer of LiF offers less impedance than its counterpart which uses a buffer layer. At room temperature, the fall of impedance is faster for the device D.

\begin{tabular}{|c|c|c|c|}
\hline Temp. & Value of Rs & Value of $\mathbf{R} \mathbf{p}$ & Value of $\mathbf{C p}$ \\
\hline $100 \mathrm{~K}$ & $20 \Omega$ & $23.35 \mathrm{~K} \Omega$ & $725 \mathrm{pF}$ \\
\hline $150 \mathrm{~K}$ & $40 \Omega$ & $13.1 \mathrm{~K} \Omega$ & $16.6 \mathrm{nF}$ \\
\hline $200 \mathrm{~K}$ & $72 \Omega$ & $7.78 \mathrm{~K} \Omega$ & $5.76 \mathrm{nF}$ \\
\hline $250 \mathrm{~K}$ & $50 \Omega$ & $5.8 \mathrm{~K} \Omega$ & $9.68 \mathrm{nF}$ \\
\hline $300 \mathrm{~K}$ & $60 \Omega$ & $3.89 \mathrm{~K} \Omega$ & $144.6 \mathrm{nF}$ \\
\hline
\end{tabular}

Table 1. Values of the Parameters in the Equivalent Circuit.

A sample computation of the parameters in the equivalent circuit of the device shown in fig. 14a is given in Table1.

\section{Encapsulation and reliability enhancement}

Ever since efficient organic light emitting diodes were reported (C.W.Tang et al,1989), there has been unending efforts for devising full color displays with the least degradation. Evolution of dark spots and consequent decay of device luminance were the reported (P.E.Burrows et al,1994) phenomena in degradation studies of organic luminescent devices. 
No doubt, the degradation due to moisture poses threat in lifetime and performance and this problem is worse in devices having flexible substrates since they are more permeable to moisture and oxygen to which organic materials are sensitive too.

The first systematic study in this respect was from Burrows et al (P.E.Burrows et al,1994) and they had proposed encapsulation as a means of circumventing the decay of life time. Large area devices when operated for extended life, there has been occurrence of short circuits. Once the device is applied with a voltage, current in the range of tens of milli amperes is allowed to send through it and short circuit begins to develop. If a high current is applied for a short period, again it causes short circuit between electrodes. The formation of microscopic conduction paths through organic layers leads to burn out when high current is applied. These paths exist initially due to the non planarity at the interfaces, eventually leading to the formation of short circuits. Considering the sustainable features of the OLED devices, the encapsulation material (G.Dennler et al,2006) should be having low moisture absorption, low curing temperature, short curing time and transparent to visible light. A structure with an encapsulation proposed by Burrows et al is shown in figure 16. The device fabricated by conventional cleaning and coating procedures to be transferred from vacuum to a glove box in nitrogen ambience. A thin bead of epoxy adhesive to be applied through syringe around the edges with care that adhesive doesn't get in contact with the active layers. A clean glass of suitable dimension to be used for covering the top and UV curing can be used to ensure proper adhesion and connections from electrodes are to be taken out with thin Au bonding connected with colloidal silver solution.

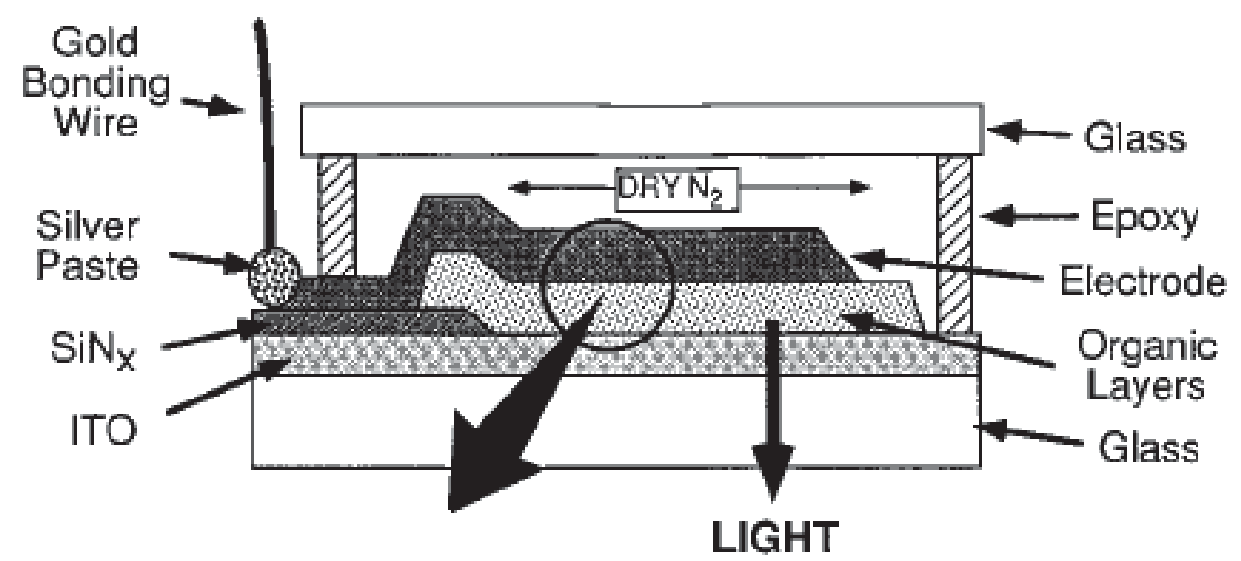

Fig. 16. Schematic on Encapsulation of OLEDs (P.E.Burrows et al,1994). 
Another method of encapsulation is based on physical lamination (Tae-Woo Lee et al,2004) of thin metal electrodes supported by elastomeric layer against an electroluminescent organic is shown in figure 17. This method relies only on van der Waals interactions to establish spatially homogeneous, intimate contacts between the electrodes and the organic layers.

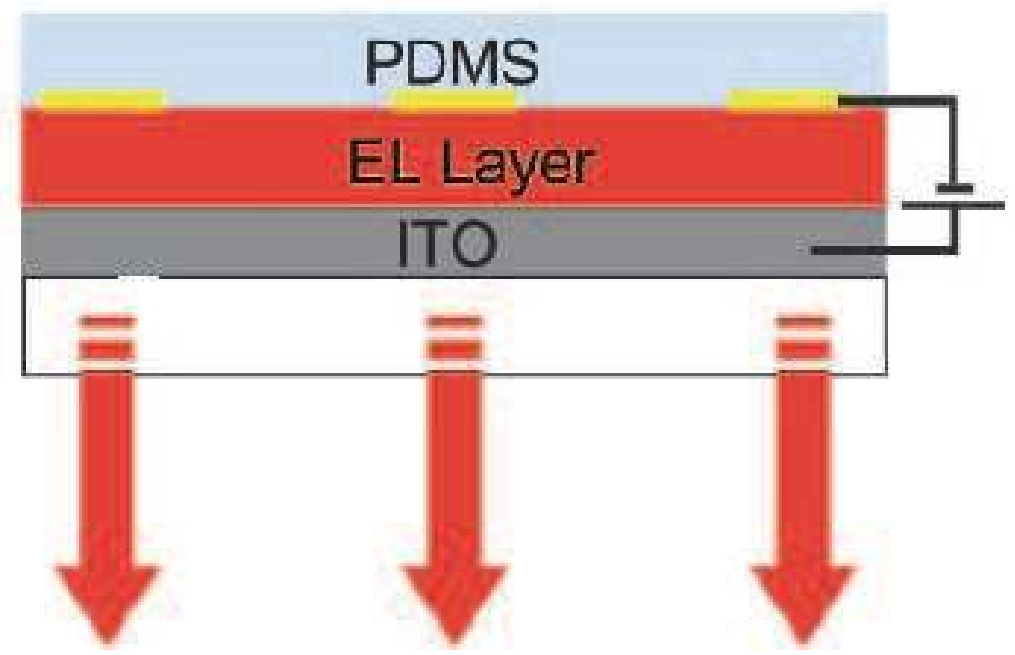

Fig. 17. Soft Contact Lamination of OLEDS [Tae-Woo Lee et al,2004).

The disruption at the electrode-organic interface can be substantially minimized with a high degree of protection against pinhole defects. This is better termed as soft contact lamination, which is intrinsically compatible with soft contact lithography which could very well be used for devices in nanometric regime. The encapsulation methods, however precise they are, induce morphological, physical or chemical changes in organic layers, which could be minimized by this soft contact lamination technique.

\section{Perimeter leakage}

Computation of leakage current is necessary for taking measures to gain control over it in order to enhance the performance of organic light emitting diodes. Not many number of studies have been reported so far in this regard and Garcia-Belmonte et al (Germa` GarciaBelmonte et al,2009) has made some pioneering works considering the structural aspects of organic light emitting diodes. No doubt, leakage current has a momentous role in the standby life of the battery operated device and hence tracing its physical origin is equally important. The ohmic behavior in reverse biased and forward biased region (till built in voltage) is assumed to be linked to leakage current component too and hence total current density can be equated as $\mathrm{J}_{\mathrm{tot}}=\mathrm{J}_{\mathrm{oper}}+\mathrm{J}_{\text {leakage }}$. The current till built in voltage has a predominant leakage component and after this point it is due to the applied potential. The surface roughness of Indium Tin Oxide Layer (K.B. Kim et al,2003) and the local damage of the organic layer induced during radio frequency sputtering of cathodes (] H. Suzuki \& M. Hikita ,2003;L.S.Liao et al,1999) are assumed to have links with the leakage paths. 


\section{References}

Amare Benor, Shin-ya Takizawa, C. Pérez-Bolivar, and Pavel Anzenbacher(2010), Energy barrier, charge carrier balance, and performance improvement in organic light-emitting diodes, Applied Physics Letters 96, 243310

C. W. Tang, S. A. VanSlyke, and C. H. Chen (1989) Electroluminescence of doped organic thin films, Journal of Applied Physics 65, 3610

G. Dennler , C. Lungenschmied , H. Neugebauer, N.S. Sariciftci , M. Latre`che ,

G. Czeremuszkin, M.R. Wertheimer(2006). A new encapsulation solution for flexible organic solar cells ,Thin Solid Films 511 - 512,349 - 353

G.G. Malliaras, J.R. Salem, P.J. Brock, J.C. Scott (1998),Current limiting mechanisms in polymer diodes, Physical Review.B 58 R13411.

G.Yu and Alan J.Heeger(1997). High efficiency photonic devices made with semiconducting polymers,Synthetic Metals 85, 1183

Germa` Garcia-Belmonte , Jose’ M. Montero , Yassid Ayyad-Limonge , Eva M. Barea ,Juan Bisquert and Henk J. Bolink(2009), Perimeter leakage current in organic light emitting diodes , Current Applied Physics 9 , 414-416

H. Suzuki, M. Hikita, (1996) Organic Light emitting diodes with radio frequency sputter deposited electron injecting eelctrodes, Applied Physics Letters 68 , 2276

H.Antoniadis. M.A.Abkowitz and B.R.Hsieh (1994), Carrier deep - trapping mobility life time products in poly (p-phenylene vinylene), Applied Physics Letters 65 (16) 2030

H.Bassler(1993). Charge Transport in Disordered Organic Photoconductors, Physica status solidi (b) $175, .15$

I.D. Parker(1994), Carrier tunneling and device characteristics in polymer light emitting diodes, Journal of Applied Physics. 75, 1656.

I.H. Campbell, T.W. Hagler, D.L. Smith, J.P. Ferraris(1996) Direct Measurement of Conjugated Polymer Electronic Excitation Energies Using Metal/Polymer/Metal Structures Physical Review Letters. 76 11,1900-1903.

J. C. Scott, J. H. Kaufman, P. J. Brock, R. DiPietro, J. Salem, and J. A.Goitia(1996), Degradation and failure of MEH-PPV light-emitting diodes, Journal of Applied Physics 79, 2745

J. H. Burroughes, D. D. C. Bradley, A. R. Brown, R. N. Marks, K. Mackay,R. H.Friend, P. L. Burns \& A.B.Holmes. (1990) Light-emitting diodes based on conjugated polymers, Nature, 347, 539 .

J.C.Scott, Philip J.Brock, Jesse R.Salem, Sergio Ramos, George G.Malliaras, Sue A .Carter and Luisa Bozano (2000). Charge transport processes in organic light emitting devices, Synthetic Metals 111-112, 289-293

J.C.Scott, S.Karg and S.A.Carter(1997). Bipolar charge and current distributions in organic lightemitting diodes Journal of Applied Physics 82(3) , 1454-60

Justin Dane and Jun Gao(2004).Imaging the degradation of polymer light emitting diodes Applied Physics Letters, 85, 3905

K.-B. Kim, Y.H. Tak, Y.-S. Han, K.-H. Baik, M.-H. Yoon, M.-H.Lee (2003). Relationship between Surface Roughness of Indium Tin Oxide and Leakage Current of Organic Light-Emitting Diode Japanese Journal of Applied Physics, Vol.42,part 2, No.4B-letters 438-440 
L. S. Roman, M. Berggren, and O. Ingana(1999).Polymer diodes with high rectiification: Applied Physics Letters. 75, 3557-3559

L.Bozano, S.A.Carter, and P.J.Brock(1998), Temperature-dependent recombination in polymer composite light-emitting diodes Applied Physics Letters 73, 3911

L.Bozano,S.A.Carter, J.C.Scott, G.G.Malliaras and P.J.Brock(1999), Temperature-and Fielddependent electron and hole mobilities in polymerlight-emitting diodes(1999),Applied Physics Letters Volume 74, Number 8

L.S. Liao, L.S. Hung, W.C. Chan, X.M. Ding, T.K. Sham, I. Bello,C.S. Lee, S.T. Lee(1999). Ionbeam-induced surface damages on tris-(8-hydroxyquinoline) aluminum, Applied Physics Letters 75, 1619.

M. A. Lampert and P. Mark(1970) Current Injection in Solids Academic, NewYork, 1970P. E. Burrows, V. Bulovic, S. R. Forrest, L. S. Sapochak, D. M. McCarty, and M. E. Thompson(1994), Reliability and degradation of organic light emitting diodes, Applied Physics Letters 65 (23)

P. S. Davids, Sh. M. Kogan, I. D. Parker, and D. L. Smith (1996). Charge injection in organic light emitting diodes: Tunneling into low mobility materials, Appl. Phys. Lett., vol. 69, pp. 2270- 2272

P.W.M.Blom and Marc J.M.de jong (1998), Electrical characterization of polymer lightemitting diodes, IEEE journal of selected topics in quantum electronics, vol. 4, no. 1

P. W. M. Blom, M. J. M. De Jong, and S. Breedijk (1997). TemperatureDependentElectronHoleRecombinationinPolymerLight-EmittingDiodes,Applied Physics Letters., vol. 71, pp. 930-932

Papadimitrakopoulos, K. Konstadinidis, T. Miller, R. Opila, E. A. Chandross and M. E. Galvin(1994).Quantum Efficiencies of Poly(Paraphenylene vinylenes), Chemistry of materials 6, 1563

S. Alem, R. de Bettignies, J. M. Nunzi, and M. Cariou(2004) Efficient polymer based interpenetrated network photovoltaic cells ,Applied Physics Letters. 84, 2178-2180

S. M. Sze(1981), Physics of Semiconductor Devices (Wiley, New York, Shun-Chi Chang and Yang Yang, Fred Wudl, Gufeng He and Yongfang Li(2001), AC impedance characteristics and modeling of polymer solution light emitting devices, Journal of physical chemistryB , 105, 11419-11423

Tae-Woo Lee and O Ok Park(2000) The Effect of Different Heat Treatments on the Luminescence Efficiency of Polymer Light-Emitting Diodes, Advancecd Materials. 12, No. 11

Tae-Woo Lee, Jana Zaumseil, Zhenan Bao, Julia W. P. Hsu, and John A. Rogers(2004) Organic light-emitting diodes formed by soft contact lamination, PNAS , 101 (2),429-433

W. H. Kim,G. P. Kushoto, H.Kim, Z.H.Kafafi(2003) Effect of annealing on the electrical properties and morphology of a conducting polymer used as anode in organic light emitting devices, Journal of Polymer Science: Part B: Polymer Physics, Vol. 41,21, 2522-2528

W.D.Gill(1972), Drift mobilities in amorphous charge transfer complexes of trinitrofluorenone and poly-n-vinylcarbazole, Journal of Applied Physics 43, 5033

Wolfgang Brutting, Stefan Berleb and Anton G.Muckl (2001). Device physics of organic lightemitting diodes based on molecular materials, Organic Electronics 2, 1-36 
Y. Cao, G. Yu, C. Zhang, R. Menon, and A.J. Heeger(1997), Polymer light emitting diodes with polyethylene dioxythiophene polystyrene sulfonate as the transparent anode,Synthetic Metals, 87,171

Y.Kawabe, M.M.Morrell, G.E.Jabbour, S.E.Shaheen, B.Kippelen and .Peyghambarian(1998) A numerical study of operational characteristics of organic light-emitting diodes.Journal of Applied Physics 84(9) 


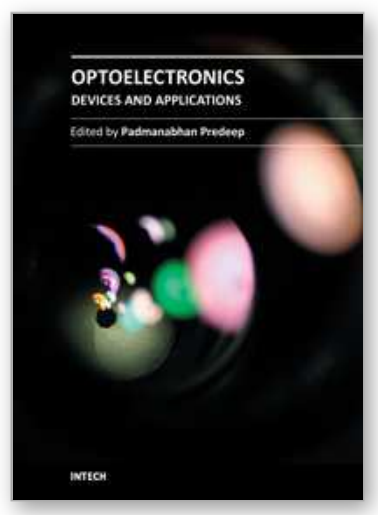

\author{
Optoelectronics - Devices and Applications \\ Edited by Prof. P. Predeep
}

ISBN 978-953-307-576-1

Hard cover, 630 pages

Publisher InTech

Published online 03, October, 2011

Published in print edition October, 2011

Optoelectronics - Devices and Applications is the second part of an edited anthology on the multifaced areas of optoelectronics by a selected group of authors including promising novices to experts in the field. Photonics and optoelectronics are making an impact multiple times as the semiconductor revolution made on the quality of our life. In telecommunication, entertainment devices, computational techniques, clean energy harvesting, medical instrumentation, materials and device characterization and scores of other areas of R\&D the science of optics and electronics get coupled by fine technology advances to make incredibly large strides. The technology of light has advanced to a stage where disciplines sans boundaries are finding it indispensable. New design concepts are fast emerging and being tested and applications developed in an unimaginable pace and speed. The wide spectrum of topics related to optoelectronics and photonics presented here is sure to make this collection of essays extremely useful to students and other stake holders in the field such as researchers and device designers.

\title{
How to reference
}

In order to correctly reference this scholarly work, feel free to copy and paste the following:

T.A. Shahul Hameed, P. Predeep, M.R. Baiju (2011). Organic Light Emitting Diodes: Device Physics and Effect of Ambience on Performance Parameters, Optoelectronics - Devices and Applications, Prof. P. Predeep (Ed.), ISBN: 978-953-307-576-1, InTech, Available from: http://www.intechopen.com/books/optoelectronics-devicesand-applications/organic-light-emitting-diodes-device-physics-and-effect-of-ambience-on-performanceparameters

\section{INTECH}

open science | open minds

\section{InTech Europe}

University Campus STeP Ri

Slavka Krautzeka 83/A

51000 Rijeka, Croatia

Phone: +385 (51) 770447

Fax: +385 (51) 686166

www.intechopen.com

\section{InTech China}

Unit 405, Office Block, Hotel Equatorial Shanghai

No.65, Yan An Road (West), Shanghai, 200040, China 中国上海市延安西路65号上海国际贵都大饭店办公楼 405 单元

Phone: +86-21-62489820

Fax: +86-21-62489821 
(C) 2011 The Author(s). Licensee IntechOpen. This is an open access article distributed under the terms of the Creative Commons Attribution 3.0 License, which permits unrestricted use, distribution, and reproduction in any medium, provided the original work is properly cited. 